\title{
Nowhere to Hide - Wide-field VLBI of the Hubble Deep Field-North
}

\author{
Jack F. Radcliffe ${ }^{* a, b, c}$ Michael A. Garrett, ${ }^{a, d}$ Thomas W. B. Muxlow, ${ }^{a}$ Robert J. \\ Beswick, $^{a}$ and Peter D. Barthel ${ }^{c}$ \\ a Jodrell Bank Centre for Astrophysics/e-MERLIN, The University of Manchester \\ M13 9PL, United Kingdom \\ ${ }^{b}$ ASTRON, the Netherlands Institute for Radio Astronomy \\ Postbus 2, 7990 AA, Dwingeloo, The Netherlands \\ ${ }^{c}$ Kapteyn Astronomical Institute, University of Groningen \\ 9747 AD Groningen, The Netherlands \\ ${ }^{d}$ Leiden Observatory, Leiden University \\ PO Box 9513, 2300 RA Leiden, The Netherlands \\ E-mail: jack.radcliffe@manchester.ac.uk
}

We present a new ultra-deep, wide-field VLBI survey targeting the central part of the Hubble Deep Field-North region using the European VLBI Network (EVN). The survey covers a large, $180 \operatorname{arcmin}^{2}$, area to milliarcsecond resolutions and microJy sensitivities. Results are presented from the first of three epochs of observations, concentrating on a new calibration technique termed multi-source self-calibration (MSSC). MSSC uses the combined response of multiple faint sources to improve the phase stability of wide-field VLBI data sets. On average, MSSC improved the signal to noise of detected target sources by $27 \%$ in naturally weighted images and $63 \%$ in uniformly weighted images when compared to phase referenced calibration. We conclude with the first science results from this survey which concentrate on the redshift $\sim 2$ sub-mm source, J123642+621331 (Waddington et al. 1999). Previous studies have indicated the presence of star-formation, together with a dusty, embedded AGN (Muxlow et al. 2005). This was confirmed by previous VLBI observations (Garrett et al. 2001, Chi et al. 2013), and makes the object a prime candidate to study the interplay between AGN and starburst activities at high redshifts. Combination imaging using the eMERGE (P.I. Muxlow) survey data (a combination of JVLA+VLA+MERLIN+eMERLIN) together with these new EVN data allows us to obtain radio images at a range of differing angular resolutions. This allows us to separate contributions from star-formation and embedded AGN.

EXTRA-RADSUR2015 (*)

20-23 October 2015

Bologna, Italy

(*) This conference has been organised with the support of the Ministry of Foreign Affairs and International Cooperation, Directorate General for the Country Promotion (Bilateral Grant Agreement ZA14GR02 - Mapping the Universe on the Pathway to SKA)

\footnotetext{
* Speaker.
} 


\section{Introduction}

Very Long Baseline Interferometry (VLBI) surveys at $\sim 1.6 \mathrm{GHz}$ can provide a dust independent tracer of accreting black holes in galaxies. VLBI typically detects bright, compact objects with brightness temperatures in excess of $10^{5} \mathrm{~K}$. In nearby galaxies, these compact objects could be a variety of sources such as Active Galactic Nuclei (AGN) or supernovae remnants. However, in distant galaxies $(\mathrm{z}>0.1)$, a VLBI detection can be reliably attributed to non-thermal AGN emission [1].

Previous, deep radio surveys have shown an upturn in the integrated source counts at sub-mJy levels. This is far in excess of those predicted by high luminosity radio galaxies and quasars that dominate at higher fluxes [2,3]. Recent multi-wavelength follow up and high resolution radio surveys, such as the eMERGE (eMERlin Galaxy Evolution) survey (P.I. Muxlow), have shown that this upturn is a mixture of active star-forming galaxies and AGN hosts. Studying the population of 'radio-quiet' AGN hosts is pivotal to our understanding of the interplay between star formation and AGN feedback at the peak star-forming era of the Universe $(\mathrm{z} \sim 2)$. Isolating the AGN can be achieved using sensitive, wide-field VLBI surveys; however, until recently, there have been many challenges preventing VLBI from being used as a survey instrument.

Originally, VLBI observations were restricted to narrow field-of-view observations that could only target a few sources near the phase centre. For wide-field VLBI to become possible, correlation has to be performed with ultra high temporal and spectral resolutions in order to restrain time and bandwith smearing away from the phase centre. In recent years, improved computational capabilities and correlator functionalities have allowed correlation to operate on feasible timescales with the required spectral and temporal resolutions. This has enabled the primary beam of a typical VLBI telescope to be imaged with minimal effects from time and bandwidth smearing. Wide-field VLBI is now a possibility and many compact sources can be detected over the course of a single observation.

The first wide-field VLBI surveys [4, 5] correlated a single phase centre with ultra high spectral and temporal resolutions in order to restrain smearing at the edge of the entire primary beam (see Fig. 1a). This method of correlation produces a single, very large, data set (often $\sim$ TB size) which increases the time taken to reduce, calibrate, and analyse the data. It has been shown that the algorithms which phase rotate data sets to other coordinates (e.g. the AIPS task UVFIX), produces significant errors which can affect the position of sources away from the phase centre [6].

In recent years, the introduction of software based correlators have established the concept of 'multiple simultaneous phase centre observing' [7, 8]. This method uses multiple phase centres with a coarser spectral and temporal resolution to produce a narrow field data set per phase centre. Phase rotation is now conducted during correlation using an accurate uv shifting algorithm [6]. Each phase centre produces a narrow field (averaged) data set that can be calibrated in an identical fashion. Compared to imaging the entire primary beam, this is both considerably less computeintensive and much more easily parallelisable. Since brute-force surveying at VLBI resolution is computationally bound, this provides a way to greatly increase the effective (computationally feasible) survey speed of VLBI observations. These phase centres can be arranged to cover as many targets as required and can be mosaicked in order to cover the entire primary beam (see Fig. 1b). 


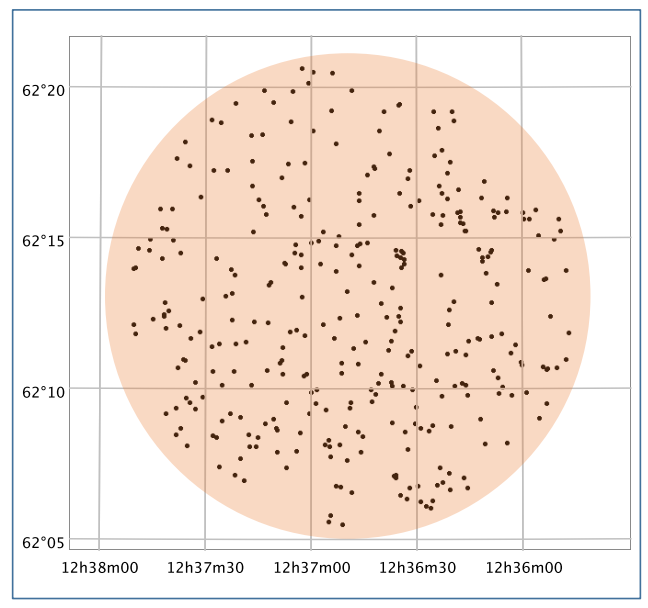

(a) Single phase centre correlation

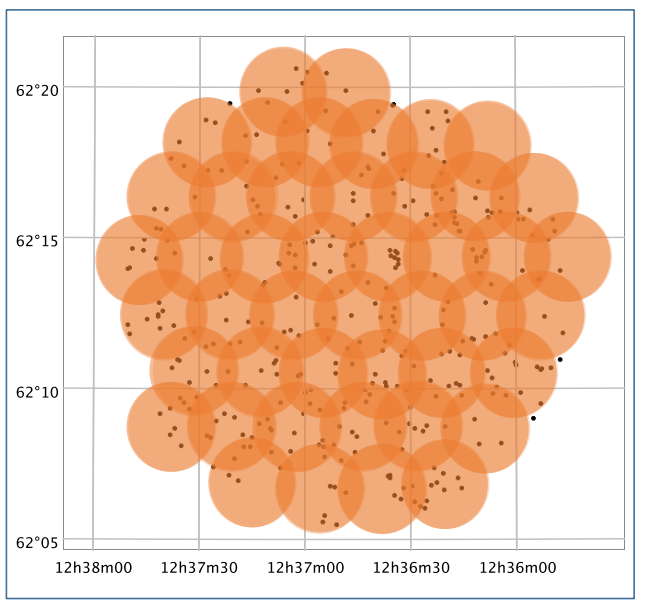

(b) Multiple phase centre correlation

Figure 1: Illustration showing the differing methods of correlating wide-field VLBI observations. The source positions shown are derived from the 1.4 GHz eMERGE data. The extent of the orange circles represent the field of view which is limited by time and bandwidth smearing. The single phase centre correlation (a), uses the pointing centre of the observations as the phase centre and correlates at a ultra high temporal and spectral resolution in order to achieve a large field of view. The multiple phase centre correlation correlates small amounts of data then shifts and averages to every phase centre specified in order to parallelise the correlation process.

In these proceedings, we present our first results from the newest, multiple phase centre correlated, wide-field VLBI observations of the Hubble Deep Field-North (HDF-N). Section 2 will summarise our observations and present our new calibration technique called multi-source selfcalibration. And in Section 3, we use multi wavelength data and combination imaging to analyse a interesting composite source that contains an AGN and signatures of star formation.

\section{Wide-field VLBI of the HDF-N}

\subsection{Observations}

We have completed the first of three, 24 hour, epochs of a $1.6 \mathrm{GHz}$ wide-field VLBI survey using the European VLBI Network (EVN). The observations target a 15 arcminute diameter area centred on the HDF-N. This survey implements the 'multiple simultaneous phase centre observing' mode of the SFXC correlator [8] in order to image a 7.5 arcminute radius area by simultaneously correlating on 582 phase centres. This allows us to achieve $\mu$ Jy r.m.s. noise levels with milliarcsecond resolutions across the whole of the primary beam. An additional 127 phase centres were used to target bright sources up to 12 arcminutes from the pointing centre. The total number of phase centres correlated is 699. The phase centres include 607 sources which have been detected in the e-MERLIN eMERGE survey [in prep.] and the VLA [9].

After standard phase referencing there were 19 sources detected $6 \sigma$ above the noise level, 18 of which are located in the central 7.5 arcminute radius area. After using a new calibration 
technique termed multi-source self calibration (MSSC), an additional source was detected, taking the total to 20 sources. This technique is described in Section 2.2.

The inner few arcminutes reach r.m.s. sensitivities of $\sim 5 \mu \mathrm{Jy} /$ beam and this is expected to reach $1 \sigma$ thermal noise levels of $\sim 1.5-4 \mu \mathrm{Jy} /$ beam (depending on telescope availability) with the addition of two further epochs. This already represents a substantial improvement when compared with the previous VLBI observations of the field which had a central r.m.s. sensitivity of 7.3 $\mu \mathrm{Jy} / \mathrm{beam}[5]$. The scientific results of this survey will be presented in a future publication.

\subsection{Multi-source Self-calibration}

VLBI observations are particularly sensitive to the temporal and spatial variations of the troposphere and ionosphere. These cause phase variations over the course of an observation. To account for these, calibration on bright, nearby and compact sources is essential. This is called 'phase referencing'. It involves employing one or more compact sources nearby (or within the primary beam of) the target field in order to correct for gain and phase fluctuations. However, many target fields do not often have compact sources which can be directly used for calibration.

We present a new calibration method termed multi-source self-calibration (MSSC). It provides an additional step to standard phase referencing. MSSC is designed to be used for multiple phase centre correlated VLBI observations but in principle, it can be used on any observation targeting multiple sources. MSSC uses multiple faint sources detected within the primary beam and combines them. The combined response of many sources across the field-of-view is generally more than sufficient to allow phase corrections to be derived. The MSSC technique is described as follows.

After standard phase referencing has been completed, each data set was re-imaged with uniform weighting and was de-convolved with the synthesised beam using the CLEAN algorithm [11]. Each set of visibilities were divided by the CLEAN model using the AIPS task UVSUB. This produced a point source with normalised amplitude, located in the centre of the target field. We note that there was small reduction in signal to noise (hereby $\mathrm{S} / \mathrm{N}$ ) when this step was undertaken. The CLEAN model cannot fully characterise the source structure and, as a result, some of the flux density is scattered into the side-lobes. Any offsets in the location of the peak brightness compared to the centroid of the phase centre were removed and the weights were adjusted with respect to the source's $\mathrm{S} / \mathrm{N}$.

The source coordinates in each data set were changed to the centre of the primary beam and the data sets were concatenated into one set using the AIPS task DBAPP. The choice of source coordinates is arbitrary. All of the source positions were changed the same coordinates so they could be stacked effectively. This resulted in a data set with visibilities that represent a normalised point source. Each baseline, time and frequency stamp now contains multiple measurements of a normalised point source. The combination of all detected sources increases the signal-to-noise and makes self-calibration possible.

The visibilities were then self-calibrated in phase using the task CALIB. A normalised point source was used as a model for just the first iteration of self-calibration. These corrections were then applied to the point source data set. The combined data set was imaged with phase corrections applied and the subsequent image was used as a model for the next round of self-calibration. This was iterated until the phase corrections converged on zero. In order to get enough signal to noise, 

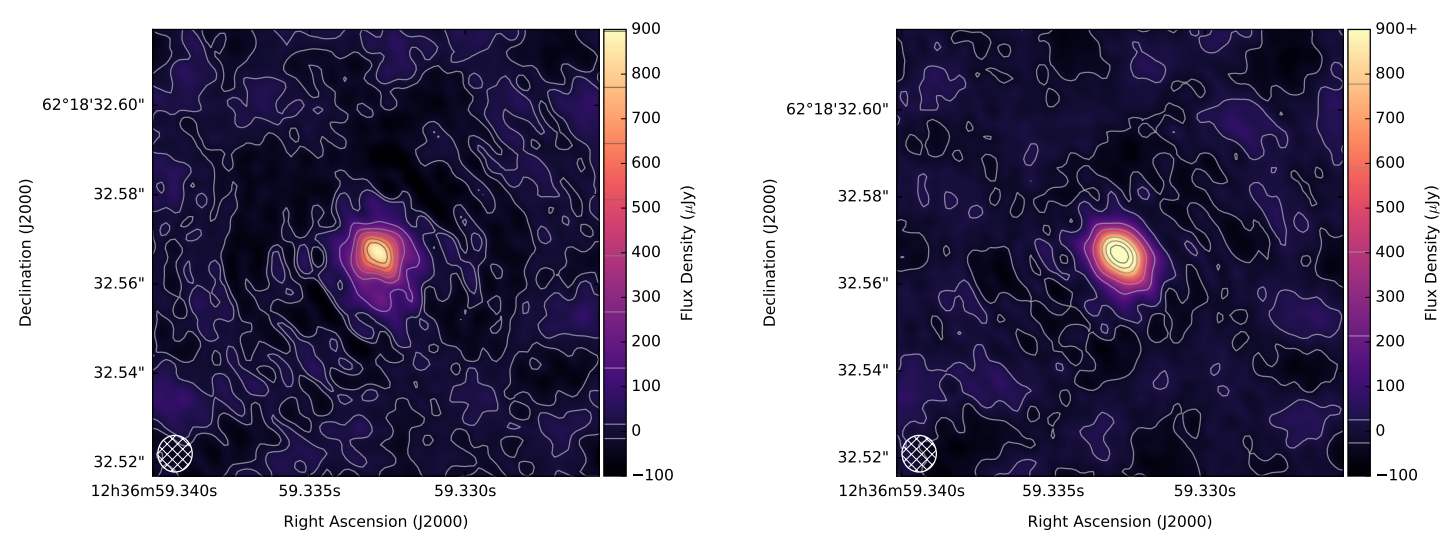

Figure 2: Compact radio source J123659+621833, which illustrates the effect MSSC has on the structure, fluxes, and noise levels achieved. The colour scale is fixed to the scale of the phasereferenced images to highlight changes in peak brightness and the noise profiles. Contours start at the noise level and are evenly spaced to the peak brightness of each image. Left panel: Source when calibrated with only standard phase referencing. This has a peak brightness of $824 \mu \mathrm{Jy} / \mathrm{beam}$, integrated flux density of $1.35 \mathrm{mJy}$ and an r.m.s. noise level of $14.7 \mu \mathrm{Jy} / \mathrm{beam}$. This gives a maximum S/N of 56.1. Right panel: The source with MSSC applied. The peak brightness is now $1.28 \mathrm{mJy} / \mathrm{beam}$, integrated flux density of $1.73 \mathrm{mJy}$ and an r.m.s. noise level of $11.1 \mu \mathrm{Jy} / \mathrm{beam}$. This results in a greatly improved S/N of 115.8 .

there is the option of combining spectral windows, polarisations or increasing the solution interval. All of these can be changed depending on the flux density distribution in the target field and the sensitivity of the observations. Phase corrections derived were written to AIPS SN tables which were attached to a dummy UV file using the task FITTP. These solution (SN) tables were then copied and applied in AIPS to all of the other phase referenced data sets. This process can be repeated if necessary.

When MSSC was compared to standard phase referencing, it was found that all sources exhibited an increase in $\mathrm{S} / \mathrm{N}$. On average, the $\mathrm{S} / \mathrm{N}$ increase was found to be $27 \%$ in naturally weighted images and $63 \%$ in the uniformly weighted images. Twelve sources can be imaged with uniform weighting compared to nine with standard phase referencing. MSSC has enabled one more source to reach the detection threshold set at $6 \sigma$. MSSC corrections also provide an improvement in the dynamic ranges and the noise profiles of the images. Figure 3 illustrates this by comparing the phase referenced set of J123658+621833 to the corresponding MSSC calibrated data. The paper describing this technique has been accepted for publication [10] and a Parseltongue script to conduct MSSC is available at https://github.com/jradcliffe5/multi_self_cal.

\section{Untangling Star Formation \& AGN - First Results}

MSSC was used on the HDF-N VLBI data set to correct for residual phase errors. This revealed a total of 20 sources. A significant population of these were found to have radio emission of 
the order 10s of $\mu \mathrm{Jy}$. This indicates that there is a significant population of radio quiet AGN. This was confirmed with e-MERLIN observations that see a population of low flux density compact objects embedded within compact star forming regions. The scientific results of this survey will be presented in a future publication. In this section, we will concentrate on one of these hybrid starburst and AGN sources called J123642+621331.

\subsection{J123642+621331}

J123642+621331 was the first source to test the new EVN data. Deep NICMOS imaging at 1.6 $\mu \mathrm{m}$ detected a very red object $\left(23.9^{m}\right)$ with a single $\operatorname{Ly}(\alpha)$ line ascribed a redshift of $z=4.424$ [12]. The source has an optical counterpart of magnitude 25 [13] and is also detected in the ISO $15 \mathrm{~m}$ and Chandra soft X-rays $[14,15]$. Interestingly, there is no SCUBA detection at $850 \mu \mathrm{m}$. This led the source to be interpreted as a dust obscured, nuclear starburst with a possible embedded AGN. There is some confusion on whether the source is actually at $\mathrm{z} \sim 4$. No direct measurement of the redshift has ever been attained. The $\operatorname{Ly}(\alpha)$ line detected was located one arcsecond to the north-west of the source. In addition, it is suggested that the object is at a redshift of 1.77 because the $\operatorname{Ly}(\alpha)$ line seen is better attributed to a [OII] 3727 line [16]. Indeed, photometric redshift estimates by Cowie (priv. comm.) also suggest that this object is at $\mathrm{z} \sim 2$. MOSFIRE observations are scheduled to directly determine the redshift of this source.

The claim of an embedded AGN were reinforced when radio observations were taken. The source has a $470 \mu \mathrm{Jy}$ VLA A array integrated flux density and has been imaged by the VLA, JVLA, MERLIN, e-MERLIN and the EVN. It was the 2001 EVN observations which first detected the radio emission of a compact component within the source [4]. The 2004 global VLBI observations revealed the existence of a a core and a small sub jet extending to the west of the source [5]. This confirms the existence of an AGN within this system. The core has a integrated flux density of $227 \pm 26 \mu \mathrm{Jy}$ and the jet has a lower integrated flux density of $121 \pm 21 \mu \mathrm{Jy}$.

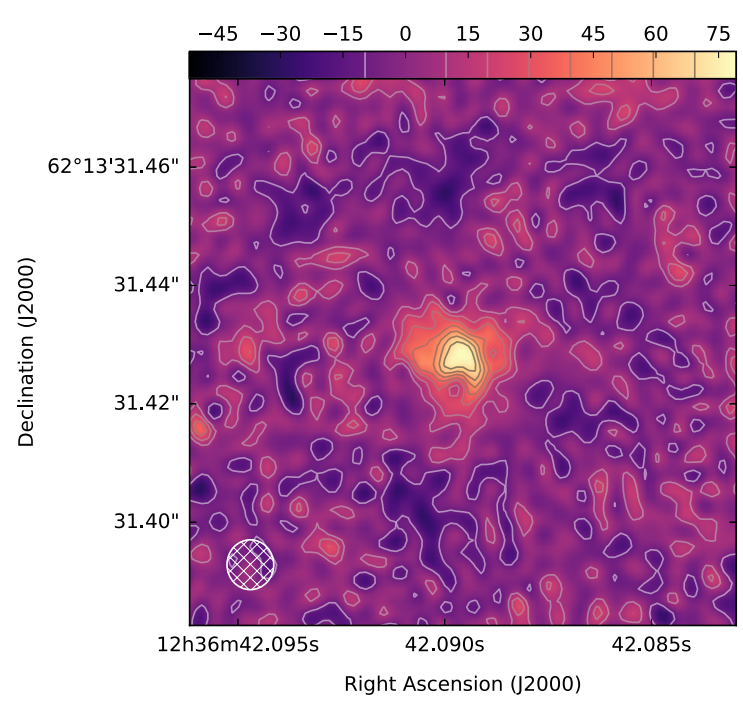

(a) $1.6 \mathrm{GHz} \mathrm{EVN}, 9 \mu \mathrm{Jy} / \mathrm{bm}$ r.m.s.

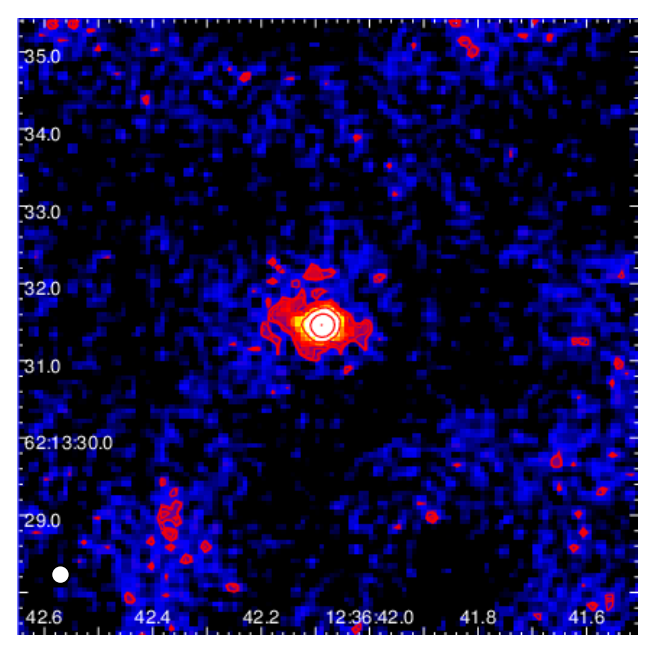

(b) $1.4 \mathrm{GHz}$ e-MERLIN, $2.5 \mu \mathrm{Jy} / \mathrm{bm}$ r.m.s.

Figure 3: Radio observations of J123642+6121331, illustrating the east-west extension suggestive of an AGN. 
The new EVN observations (2014) also detected this source and showed a core with a small east-west sub-jet extension, as seen in the global VLBI observations (see Fig. 3a). This acted as confirmation that the calibration was correct. Interestingly, the core of the object shows a 50\% decrease in integrated flux density with respect to the global VLBI observations. The core has a integrated flux density of $123 \pm 26 \mu \mathrm{Jy}$ and the jet has an integrated flux density of $136 \pm 30 \mu \mathrm{Jy}$. This intrinsic variability is typical behaviour of an AGN and further confirms the existence of an embedded AGN within this system. An extension to this jet of angular size of 0.7 arcsec is also shown in the e-MERLIN L-band image (see Fig. 3b). e-MERLIN reveals an additional extension emanating from west to east which is not seen in global VLBI. The new EVN observations show a hint $(3.2 \sigma)$ of the second sub-jet from the west to east which is apparent on e-MERLIN baselines. The diffuse emission shown in the EVN observations suggest that there are hints of starburst activity out to $\sim 7 \mathrm{pc}$ from the AGN core. This would explain the extremely red character in the IR and confirm initial suspicions of a nuclear starburst. However, this is only a $3 \sigma$ detection and more epochs would be needed to evaluate this claim.

\subsection{Combination Imaging}

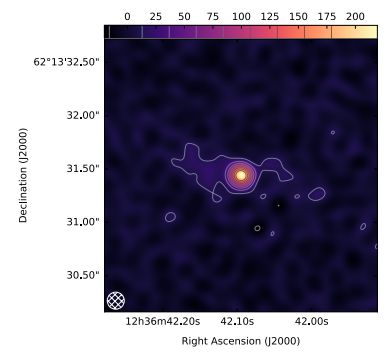

(a) 150mas beam

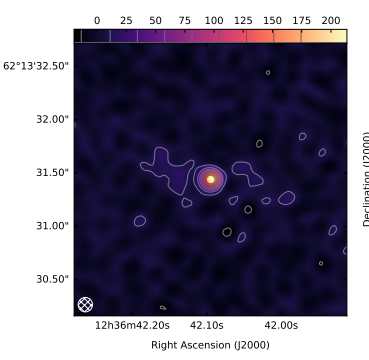

(b) 100mas beam

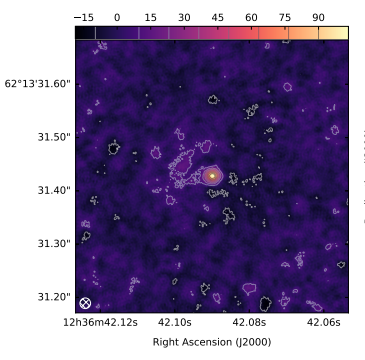

(c) 25 mas beam

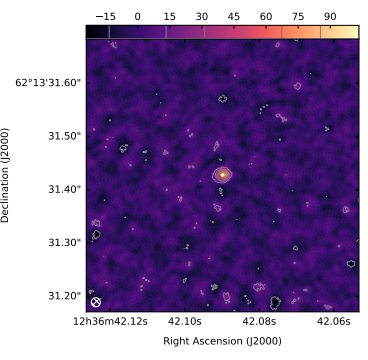

(d) 10 mas beam

Figure 4: Combined e-MERLIN and EVN VLBI data weighted to achieve multiple resolution images of $\mathrm{J} 123642+621331$. The colour scale is in $\mu \mathrm{Jy} / \mathrm{bm}$ and milliarcsecond has been shortened to mas.

Combining data from various radio telescope arrays can be a very powerful tool in understanding the development of structure at various angular scales (e.g. [13], [17]). The HDF-N field has a wealth of data at both $1.4 \mathrm{GHz}$ and $5 \mathrm{GHz}$ which can be used in combination imaging. Our goal is to combine VLA, JVLA, MERLIN, e-MERLIN, EVN and global VLBI data to observe how source structure changes from arcsecond to milliarcsecond resolutions. Understanding structure at various angular scales could prove to be key in understanding the relationship between star formation and AGN in these distant objects.

In our example, we combined L Band e-MERLIN and EVN data to investigate the source J123642+621331 (see Fig. 4). This set has been multiply weighted with respect to UV distance in order to attain multiple images at different angular resolutions. Fig. 4a shows the bright core, the east to west jet seen in the eMERLIN observations, and the corresponding extension from west to east. As the resolution increases, the west to east extension has disappeared. This could be due to the reduction of sensitivity at these intermediate resolutions, large scale structure being resolved out or AGN jet beaming. By Fig. 4c, the east to west jet show signs of a possible radio hotspot or compact star forming regions in the areas with higher flux densities. As we reach $\sim 10$ 
millarcsecond resolutions (Fig. 4d), only the embedded, compact AGN core is still visible and any large scale structure has been resolved out.

\section{Conclusions}

We presented the first results of our EVN, wide-field VLBI observations. These observations completely mapped a 7.5 arcminute radius area, centred on the HDF-N, to milliarcsecond resolutions and microJy sensitivities. We introduced a new calibration technique, termed multi-source self-calibration (MSSC), which uses a combination of multiple faint target sources in order to correct for residual phase errors. With standard phase referencing, 19 sources were detected in this field but, with MSSC corrections applied, one extra source was detected taking the total to 20. On average, MSSC greatly improves the $\mathrm{S} / \mathrm{N}$ of detected sources by $63 \%$ in uniformly weighted images, and $27 \%$ in naturally weighted images. A large proportion of these sources were found to have integrated flux densities of the order a few $10 \mathrm{~s}$ of $\mu \mathrm{Jy}$. This is typical of radio weak AGN hosts in star-forming galaxies.

To conclude, we focused on one of these hybrid AGN-starforming systems. J123642+621331 is at a redshift of $\sim 2$, and shows signs of both star-formation (extremely red object in the IR) and AGN activity. The new EVN observations confirmed the existence of the AGN, as previously detected by previous VLBI observations [4, 5], and also showed a small sub-jet extension. We explored J123642+621331 using combination imaging in order to see the evolution of structure at many physical scales using e-MERLIN and EVN data. Using this we managed to gain an insight into the interplay between the embedded AGN and compact star formation in this object. The scientific results of this survey will be presented in a future publication.

\section{References}

[1] L. Kewley et al., Compact radio emission from warm infrared galaxies, The Astrophysical Journal 5302 p704 (2000).

[2] I. Prandoni et al., The ATESP radio survey III., Astronomy \& Astrophysics 365392 (2001), [arXiv:astro-ph/0010444].

[3] M. Huynh et al., Radio observations of the hubble deep field-south region. II. The $1.4 \mathrm{GHz}$ catalog and source counts, The Astronomical Journal 1304 p1373 (2005), [arXiv: astro-ph/ 0506047 ].

[4] M. A. Garrett et al., AGN and starbursts at high redshift: High resolution EVN radio observations of the Hubble Deep Field, Astronomy \& Astrophysics 366 L5-L8 (2001), [ast ro-ph/ 0102037 ].

[5] S. Chi et al., Deep, wide-field, global VLBI observations of the Hubble deep field north (HDF-N) and flanking fields (HFF), Astronomy \& Astrophysics 550 A68 (2013), [arXiv: 1301.1253].

[6] J. Morgan et al., VLBI imaging throughout the primary beam using accurate UV shifting, Astronomy \& Astrophysics 526 A140 (2011), [arXiv: 1302.6040$]$.

[7] A. T. Deller et al., DiFX-2: a more flexible, efficient, robust, and powerful software correlator, Publications of the Astronomical Society of the Pacific 123901 p275-287 (2011), [arXiv:1101.0885].

[8] A. Keimpema et al., The SFXC software correlator for very long baseline interferometry: algorithms and implementation, Experimental Astronomy 392 pp.259-279 (2015), [arXiv: 1502.00467 ]. 
[9] G. Morrison et al., Very Large Array $1.4 \mathrm{GHz}$ Observations of the GOODS-North Field: Data Reduction and Analysis, The Astrophysical Journal Supplement Series 1881 p178, [arXiv: 1004.1671$]$.

[10] Radcliffe et al., Multi-source self-calibration: Unveiling the microJy population of compact radio sources, accepted Astronomy \& Astrophysics, [arXiv: 1601.04452$].$

[11] B. G. Clark, An efficient implementation of the algorithm 'CLEAN', Astronomy \& Astrophysics 89 p377 (1980).

[12] I. Waddington et al., NICMOS imaging of the dusty microjansky radio source VLA J123642+621331 at $z=4.424$, The Astrophysical Journal Letters 5262 L27 (1999), [arXiv: astro-ph/9910069].

[13] T. W. B. Muxlow et al., High-resolution studies of radio sources in the Hubble Deep and Flanking Fields, 3584 p1159-1194 (2005), [arXiv:astro-ph/0501679].

[14] H. Aussel et al., ISOCAM observations of the Hubble Deep Field reduced with the PRETI method, Astronomy \& Astrophysics 342 p313-336 (1999), [astro-ph/ 9810044 ].

[15] W. N. Brandt et al., The Chandra deep survey of the Hubble Deep Field North Area. IV. An ultradeep image of the HDF-N, The Astronomical Journal 1221 p1 (2001), [arXiv:astro-ph/0102411].

[16] G. Hasinger, Absorption properties and evolution of active galactic nuclei, Astronomy \& Astrophysics 4903 p905-922 (2008), [arXiv: 0808 .0260].

[17] D. Fenech et al., Wide-field Global VLBI and MERLIN combined monitoring of supernova remnants in M82, MNRAS 4081 p607-621 (2010), [arXiv: 1006.1504 ].... 